\title{
Development of a disease-specific graded prognostic assessment index for the management of sarcoma patients with brain metastases (Sarcoma-GPA)
}

\author{
Anna Patrikidou ${ }^{1,2^{*}}$, Loic Chaigneau ${ }^{3}$, Nicolas Isambert ${ }^{4}$, Kyriaki Kitikidou $^{5}$, Ryan Shanley ${ }^{6}$, Isabelle Ray-Coquard ${ }^{7}$, \\ Thibaud Valentin ${ }^{8}$, Bettina Malivoir ${ }^{9}$, Maryline Laigre ${ }^{10}$, Jacques-Olivier Bay ${ }^{11}$, Laurence Moureau-Zabotto ${ }^{12}$, \\ Emmanuelle Bompas ${ }^{13}$, Sophie Piperno-Neumann ${ }^{14}$, Nicolas Penel ${ }^{15}$, Thierry Alcindor ${ }^{16}$, Cécile Guillemet ${ }^{17}$, \\ Florence Duffaud ${ }^{18}$, Anne Hügli ${ }^{19}$, Cécile Le Pechoux ${ }^{1}$, Frédéric Dhermain ${ }^{1}$, Jean-Yves Blay ${ }^{7}$, Paul W. Sperduto ${ }^{6}$ and \\ Axel Le Cesne ${ }^{1}$
}

\begin{abstract}
Background: Brain metastases from sarcomatous lesions pose a management challenge owing to their rarity and the histopathological heterogeneity. Prognostic indices such as the Graded Prognostic Assessment (GPA) index have been developed for several primary tumour types presenting with brain metastases (e.g. lung, breast, melanoma), tailored to the specifics of different primary histologies and molecular profiles. Thus far, a prognostic index to direct treatment decisions is lacking for adult sarcoma patients with brain metastases.
\end{abstract}

Methods: We performed a multicentre analysis of a national group of expert sarcoma tertiary centres (French Sarcoma Group, GSF-GETO) with the participation of one Canadian and one Swiss centre. The study cohort included adult patients with a diagnosis of a bone or soft tissue sarcoma presenting parenchymal or meningeal brain metastases, managed between January 1992 and March 2012. We assessed the validity of the original GPA index in this patient population and developed a disease-specific Sarcoma-GPA index.

Results: The original GPA index is not prognostic for sarcoma brain metastasis patients. We have developed a dedicated Sarcoma-GPA index that identifies a sub-group of patients with particularly favourable prognosis based on histology, number of brain lesions and performance status.

Conclusions: The Sarcoma-GPA index provides a novel tool for sarcoma oncologists to guide clinical decisionmaking and outcomes research.

Keywords: Sarcoma, Brain metastasis, Prognostic index

\section{Background}

Brain metastasis $(\mathrm{BM})$ in adult sarcoma patients is a rare occurrence [1-3]. Owing to this rarity, little formal exploration exists in the literature, and evidence-based data is scant. In contrast, BM management in other cancer types has recently evolved in part due to advances in

\footnotetext{
* Correspondence: anna.patrikidou@sarahcannonresearch.co.uk

${ }^{1}$ Gustave Roussy Cancer Campus, Villejuif, France

2Present Address: Sarah Cannon Research Institute and UCL Cancer Institute \& University College London Hospitals, 93 Harley Street, London W1G 6AD, UK

Full list of author information is available at the end of the article
}

imaging and treatment but also because of the progressive development of prognostic indices.

Whereas in the past, it was believed that all patients with brain metastases had a grim prognosis, we now know that this patient population is markedly heterogeneous and prognosis varies widely. A number of prognostic indices were developed in order to guide treatment decisions, notably the RTOG (Radiation Therapy Oncology Group) Recursive Partitioning Analysis (RPA) [4, 5], the Score Index for radiotherapy (SIR) [6] and the Basic Score for Brain Metastases (BSBM) [7]. A more recent index, the Graded Prognostic Assessment (GPA) index [8], Table 1)

(c) The Author(s). 2020 Open Access This article is distributed under the terms of the Creative Commons Attribution 4.0 International License (http://creativecommons.org/licenses/by/4.0/), which permits unrestricted use, distribution, and 
Table 1 Original Graded Prognostic Assessment (GPA) score

\begin{tabular}{|c|c|c|c|}
\hline \multirow[b]{2}{*}{ Prognostic Factor } & \multicolumn{3}{|c|}{ Graded Prognostic Assessment (GPA) Scoring Criteria ${ }^{a}$} \\
\hline & 0 & 0.5 & 1.0 \\
\hline Age, years & $>60$ & $50-60$ & $<50$ \\
\hline KPS & $<70$ & $70-80$ & $90-100$ \\
\hline ECM & Present & - & Absent \\
\hline Number of BM & $>3$ & $2-3$ & 1 \\
\hline
\end{tabular}

KPS Karnofsky performance status, ECM extracranial metastasis, $B M$ brain metastases

${ }^{a}$ As per Sperduto et al 2008 Int J Radiation Oncol Biol Phys

was developed to address the limitations of previous indices, utilizing knowledge on the prognostic value of the number of brain metastases and shaping the index so as to guide treatment decisions rather than to reflect treatment results. Comparison with previous indices has indicated its improved utility and prognostic power [8]. The original GPA was validated and refined with disease-specific prognostic indices for the major types of cancer that develop brain metastases, such as breast, lung, melanoma, renal and gastrointestinal cancer $[9,10]$ and has evolved to incorporate information on histotype $[11,12]$ and tumour molecular characteristics [13, 14]. Population-based reports have confirmed the prognostic significance of histotype in breast cancer for the predilection of site of distant metastasis and the development of brain metastases $[15,16]$.

Prognosis of brain metastases is not uniform throughout the different forms of cancer, nor amongst patients suffering from the same cancer type. This knowledge also implies that use of the same treatment for all patients and all primary types for the management of brain metastases is not appropriate, especially in the face of recent developments of treatment modalities.

The one-size-fits-all treatment paradigm that is no longer appropriate in other cancer types is still dominating the management of sarcoma patients with brain metastases.

Brain metastases in sarcoma patients is rather rare, with a reported incidence of $<1$ to $8 \%$. The French Sarcoma Group (GSF-GETO) has recently published the largest series to date of sarcoma patients with brain metastases, describing their characteristics, treatment modalities, prognostic factors and outcome [17]. This report identified leiomyosarcoma and liposarcoma as the most frequent histologies in sarcoma BM patients, and identified several characteristics of long survivors (younger age, unique lesions, lower grade tumors, better PS, longer time to development of brain metastases, higher use of local treatment modalities) [17].

On the basis of this cohort, we aimed to (a) assess the validity of the original GPA index in sarcoma patients with brain metastases, and (b) develop an informative, sarcoma-specific GPA index (Sarcoma-GPA), to serve as a prognostic index for treatment decisions and outcomes analyses.

\section{Methods}

Patient cohort and data collection

Under the auspices of GSF-GETO, a project involving a multi-institutional retrospective analysis project of sarcoma patients with brain metastases (cerebral or meningeal lesions) was developed (BRAINSARC) [17]. Institutional ethics committee approval was obtained for each centre. The database included patients from 15 French, one Swiss and one Canadian centre. The retrospective data collection was limited to patients managed between January 1992 and March 2012, to ascertain homogeneity in histological diagnosis and classification, namely uniform use of the FNCLCC grading system [18], and to ensure adequate follow-up. The results of this analysis are published elsewhere [17]. Utilizing and enriching this GSF-GETO database, we developed the current project of implementation of the original GPA on sarcoma patients and development of a disease-specific index (Sarcoma-GPA).

Data collection procedures for the BRAINSARC project are described in detail elsewhere [17]. Specifically for the current project, data collection was completed, verified and annotated for the GPA components, notably age at BM diagnosis, Karnofsky performance status (KPS), number of brain lesions and presence of extracranial metastases (ECM), as well as for overall survival (OS). For the development of the disease-specific GPA index, data on ECOG performance status, localization of brain metastasis, time to brain metastasis (TTBM), site of ECM, histological subtype and grade were also collected, verified and annotated. For the histological classification, the 2013 WHO Classification of Tumors of Soft Tissue and Bone was used [19].

\section{Statistical analysis}

Overall survival was estimated from the time of BM diagnosis to the date of death or last follow-up. TTBM was estimated from initial sarcoma diagnosis to the time of BM diagnosis.

For the implementation of the GPA index on our sarcoma cohort, data for each of the four index components 
were coded according to the original GPA score [8] (Table 1). Each patient was attributed an overall score corresponding to the sum of the scores of individual index components. The GPA index was analyzed in four levels, as per the original description, with group cut-offs of $0-1$, $1.5-2.5,3$ and 3.5-4. The GPA scores were subsequently correlated with OS. Survival distributions for individual variables but also for each individual index level compared with all other levels were compared with the log-rank and Mann-Whitney tests using a significance level of 0.001 . Overall survival curves for each level of the GPA index were estimated using the Kaplan-Meier method, using the same significance level.

For the development of the Sarcoma-GPA index, cutoffs were decided based on previous GPA indices and on biological sense. Given that the study aim was to identify a meaningful, prognostic way of separating patient subgroups in terms of prognosis, in some instances different variations of cut-offs were attempted in order to identify significant, meaningful cut-offs. Prognostic factors for survival were analyzed by two methods: multivariate Cox regression (MCR) and recursive partitioning analysis (RPA). RPA aided in the identification of best splitting rules amongst prognostic factors. This dual MCR-RPA methodology has been previously shown to be an effective tool in the design of prognostic indices [10, 11, 20]. Prognostic factors found to be significant by either method were used to develop and refine the final Sarcoma-GPA index. Optimal cut-offs for groups were chosen to be consistent with previous disease-specific GPA literature (group cut-offs 0-1, 1.5-2, 2.5-3 and 3.5-4), weighing the significant factors in proportion to the magnitude of the hazard ratio such that 4.0 is the best and 0.0 is the worst $[6,10,11,13,14]$. Multivariate analysis was performed using the Cox proportional hazards model. Analyzed variables were age, KPS, ECOG PS, sarcoma type (bone versus soft tissue), localization, tumor size, histological grade and type, time to first metastasis, time to brain metastasis, TTBM, BM lesion number and localisation, presence of and type of ECM at the time of BM diagnosis, and all possible two-way interactions. For hazard ratios, the reference category is defined to have a $H R=1, H R>1$ indicating a higher death rate compared to the reference category. The univariate and multivariate analyses were performed separately for the ECOG PS and KPS variables, as these represent the same clinical characteristic (patient general functional status), in an attempt to identify any clinically pertinent difference in their use within the prognostic score. Since the objective was to develop a prognostic index to guide treatment, no treatment-related variable was analyzed. A forward selection procedure with a cutoff $p$-value of 0.05 was used to establish the initial model.

For the development of the final model, if individual classes within the investigated variables failed to show statistically significant differences of survival, groupings of multiple levels with similar outcomes were explored. Prognostic factors found to be significant by either MCR or RPA were retained in the final MCR model in order to improve its prognostic ability.

In the final Sarcoma-GPA index, a score of 4 correlates with the best prognosis and a score of 0 with the worst. The Kaplan-Meier method was used to estimate the survival curve for each prognostic group. The logrank and Mann-Whitney test for censored data were used to test for significant survival differences amongst levels of the Sarcoma-GPA index (statistical significance defined as $p<0.001)$. The goodness of fit was evaluated using the Harrell's concordance index (c-index), using 200 bootstrap replications to estimate out-of-sample performance, as well as ROC (Receiver Operating Characteristic) analysis. The final Sarcoma-GPA index was chosen as a balance of performance metrics and simplicity.

Analysis was performed using the SPSS Statistics ver-

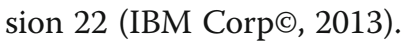

The development of the sarcoma-specific index was done in collaboration with the team that described the original and disease-specific GPA indices.

\section{Results}

A total of 251 patients with BMs (parenchymal, meningeal and combination of such lesions) of a sarcoma primary fulfilling the study criteria were included in the final analysis (5 patients that were excluded from the initially reported analysis owing to missing data were included in the current analysis as data were retrieved through a second, project-specific data collection as described above). The patient and disease characteristics are shown in Table 2, consistent to what has been previously reported [8]. Median follow-up was 2.79 months (OS range: $0.06-$ 133.02 months). The median overall survival was 3.160 months. Presence of ECM was predominant at the time of BM diagnosis (91\%), median TTBM was 18.5 months, whilst median time from first metastasis to development of BM (TMtBM) was 9.6 months. Treatment modalities details are presented in Additional file 1: Table S1.

\section{Implementation of the original GPA score in sarcoma patients}

The application of the original GPA score in our sarcoma patient cohort did not allow for validation of its prognostic value. The differences in median OS for each GPA index level were not significant for clear discrimination between each subgroup, especially for the higherscoring subgroups (Additional file 3: Figure S1). Of the individual index components using score-specific cutoffs, the KPS was the most highly significant, showing the best discrimination amongst component levels $(p<$ 0.001) (Additional file 4: Figure S2). 
Table 2 Cohort characteristics.

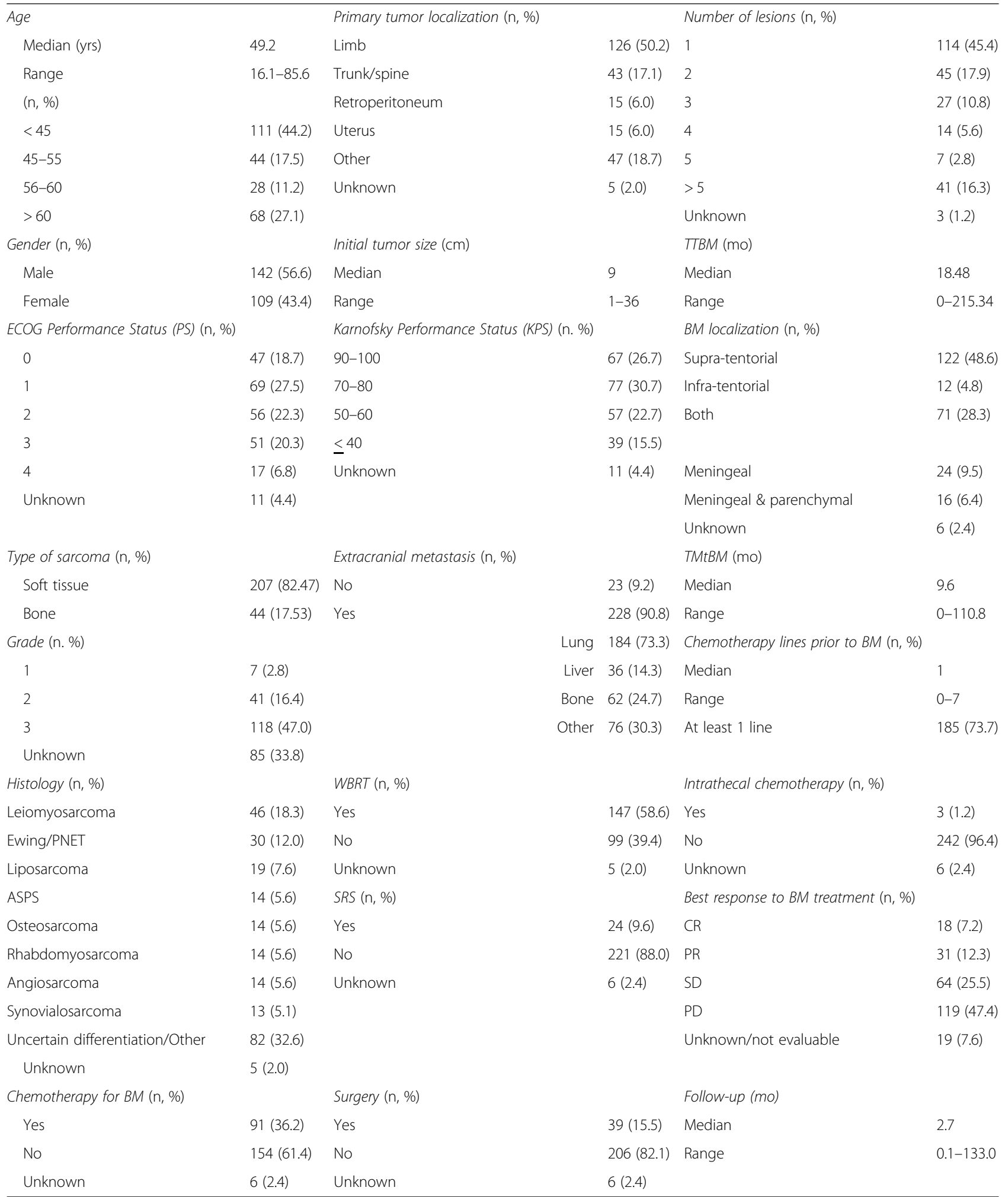

ASPS Alveolar soft part sarcoma, $B M$ brain metastasis, $\mathrm{cm}$ centimeters, $C R$ complete response, ECOG Eastern Cooperative Oncology Group, KPS Karnofsky performance status, mo months, $n$ number, $O S$ overall survival, $P D$ progressive disease, $P N E T$ primitive neuroectodermal tumour, $P R$ partial response, $P S$ performance status, SD stable disease, SRS stereotactic radiosurgery, TMtBM time from first metastasis to brain metastasis, TTBM time to brain metastasis, yrs. years, WBRT whole brain radiotherapy 


\section{Development of the sarcoma-GPA index}

Different variable cut-offs were individually assessed for significance in regard with overall survival, and were subsequently tested in the under development index. The variables identified as significant in the univariate (age, histology, number of CNS metastases, ECOG PS, KPS, TTBM) and multivariate analysis (histology, number of CNS metastases, ECOG PS, KPS) were individually assessed for the index (Fig. 1, Table 3). RPA analysis results were consistent with the MCR analysis, identifying the number of BMs and the ECOG PS as predictive for survival (Fig. 2).

For the development of the sarcoma-specific GPA, the variables and respective cut-offs identified as significant were tested in different combinations. The best performing split levels and groups, as indicated by both MCR and RPA, lead to the identification of the optimal SarcomaGPA index, that included the three variables retained as significant: histology, number of CNS metastases and performance status (Fig. 3). The final index used 4-point cutoffs for the prognostic group levels (scores 0-1, 1.5-2.0,
2.5-3 and 3.5-4), consistent with previously reported GPA scores, with the GPA1 group (score $0-1$ ) having the worst prognosis and the GPA4 group (score 3.5-4) having the better prognosis (Fig. 3). The spit levels chosen for the Sarcoma-GPA index for the histology variables were as follows: group H1 $(n=22$; adipocytic tumors, including liposarcoma and myxoid liposarcoma), group $\mathrm{H} 2(n=111$; smooth muscle tumors including leiomyosarcoma; skeletal muscle tumours including rhabdomyosarcoma; chondroosseous tumors including osteosarcoma; fibroblastic/myofibroblastic tumors including fibrosarcoma; so-called fibrohistiocytic tumors including pleiomorphic MFH"/ undifferentiated pleiomorphic sarcoma; "vascular tumors, including angiosarcoma; tumors of uncertain differentiation including intimal sarcoma), group $\mathrm{H} 3(n=89$; tumors of uncertain differentiation, including synovial sarcoma, clear cell sarcoma, epithelioid sarcoma, small round cell tumors, undifferentiated sarcomas, and also malignant peripheral nerve sheath tumor/neurofibrosarcoma and one case of phyllodes tumor/cystosarcoma of the breast) and group H4 [ $n=24$; predominantly alveolar soft part sarcomas
A
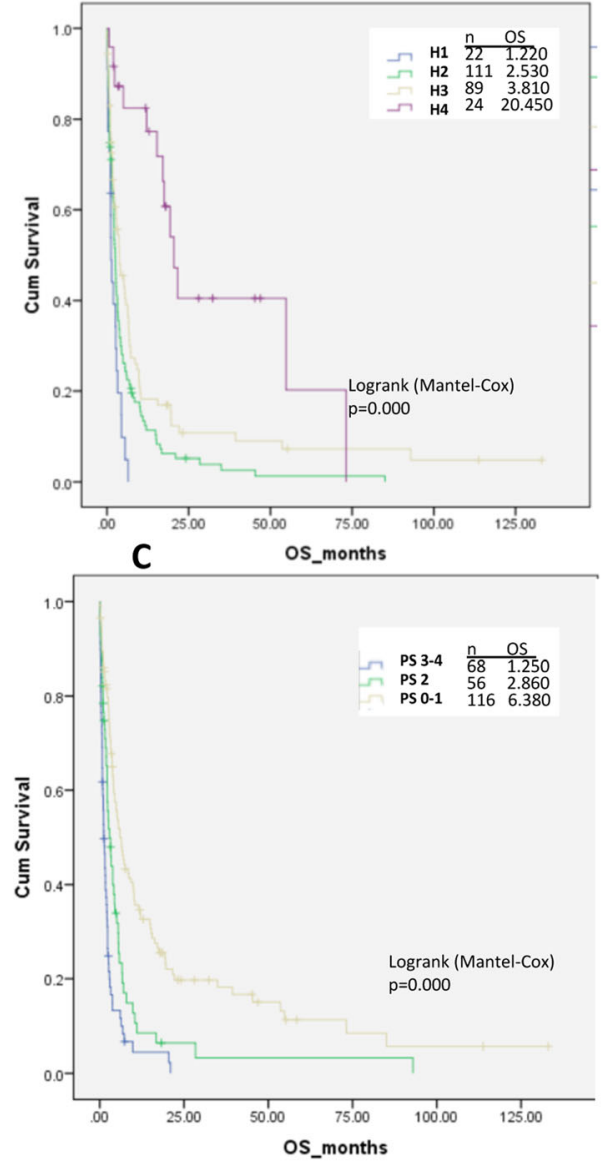

B
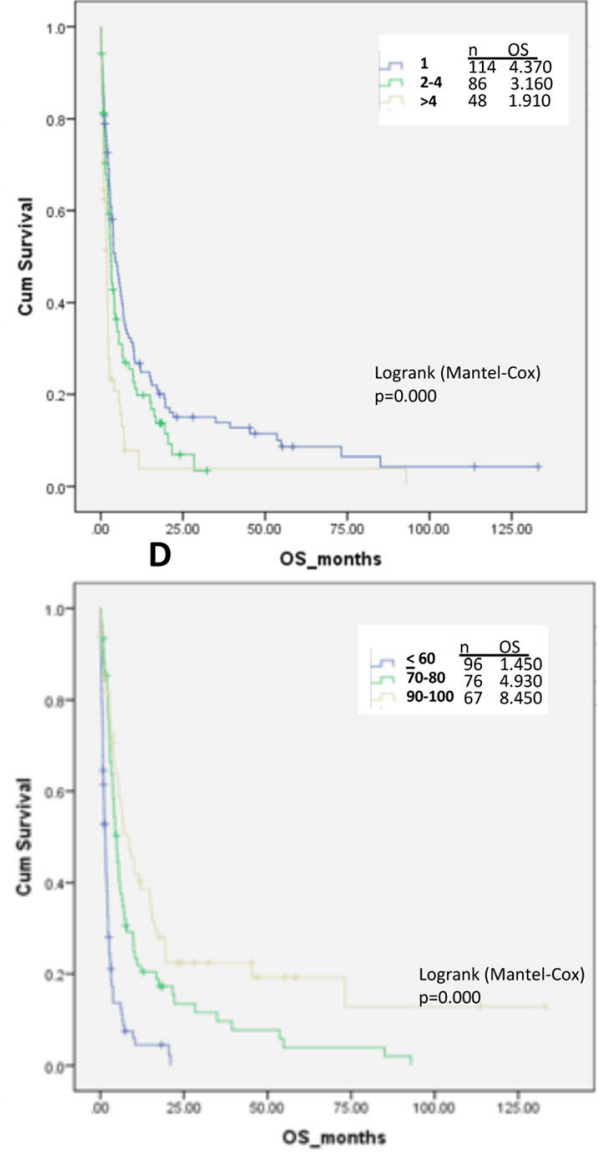

Fig. 1 Significant variables in multivariate analysis. a: Histology; b: number of CNS metastases; c: ECOG performance status (PS); d: Karnofsky performance status (KPS). CNS: central nervous system; H1-H4: histology groups (see text for description); OS: overall survival 
Table 3 Univariate and multivariate analyses

\begin{tabular}{|c|c|c|c|c|c|c|c|c|}
\hline \multirow[t]{3}{*}{ Variable } & \multicolumn{4}{|l|}{ UVA } & \multicolumn{4}{|l|}{ MVA } \\
\hline & \multirow[t]{2}{*}{$p$-value } & \multirow[t]{2}{*}{$H R$} & \multicolumn{2}{|l|}{$95 \% \mathrm{Cl}$} & \multirow[t]{2}{*}{$p$-value } & \multirow[t]{2}{*}{$\mathrm{HR}$} & \multicolumn{2}{|l|}{$95 \% \mathrm{Cl}$} \\
\hline & & & Lower & Upper & & & Lower & Upper \\
\hline \multicolumn{9}{|l|}{ Age (reference: > 55) } \\
\hline Age [45-55] & .209 & .765 & .503 & 1.162 & .099 & .685 & .436 & 1.074 \\
\hline Age $<45$ & .003 & .646 & .483 & .863 & .802 & .957 & .677 & 1.352 \\
\hline Gender & .620 & .934 & .714 & 1.222 & & & & \\
\hline Sarcoma_type (bone vs soft tissue) & .701 & .936 & .667 & 1.312 & & & & \\
\hline \multicolumn{9}{|l|}{ Histology (reference: H1) } \\
\hline Histology: H2 & .016 & .557 & .346 & .896 & .006 & .493 & .297 & .819 \\
\hline Histology: H3 & .000 & .381 & .231 & .627 & .000 & .334 & .192 & .581 \\
\hline Histology: H4 & .000 & .129 & .063 & .263 & .000 & .159 & .072 & .349 \\
\hline \multicolumn{9}{|l|}{ Grade (reference: 3) } \\
\hline Grade $=2$ & .157 & .756 & .513 & 1.114 & & & & \\
\hline Grade $=1$ & .174 & .561 & .244 & 1.291 & & & & \\
\hline Extracranial metastases (no, yes) & .679 & .907 & .571 & 1.441 & & & & \\
\hline Extracranial metastases: Lung (no, yes) & .621 & 1.079 & .798 & 1.458 & & & & \\
\hline Extracranial metastases: Liver (no, yes) & .521 & .881 & .598 & 1.297 & & & & \\
\hline Extracranial metastases: Bone (no, yes) & .563 & 1.099 & .798 & 1.513 & & & & \\
\hline Extracranial metastases: other (no, yes) & .291 & .853 & .636 & 1.145 & & & & \\
\hline \multicolumn{9}{|l|}{$\begin{array}{l}\text { Number of CNS metastases } \\
\text { (reference: }>4 \text { ) }\end{array}$} \\
\hline Number of CNS metastases (2-4) & .007 & .590 & .402 & .866 & .092 & .699 & .462 & 1.060 \\
\hline Number of CNS metastases (1) & .000 & .453 & .314 & .654 & .022 & .620 & .412 & .933 \\
\hline \multicolumn{9}{|l|}{ ECOG PS (reference: 3 or 4) } \\
\hline$E C O G P S=2$ & .001 & .523 & .359 & .761 & .000 & .468 & .314 & .696 \\
\hline PS (0 or 1) & .000 & .274 & .195 & .384 & .000 & .305 & .211 & .441 \\
\hline \multicolumn{9}{|l|}{ Localisation (reference: supra-tentorial) } \\
\hline Localisation: infra-tentorial & .317 & .763 & .450 & 1.295 & & & & \\
\hline Localisation: supra-tentorial \& infra-tentorial & .887 & 1.057 & .490 & 2.282 & & & & \\
\hline Localisation: meningeal & .120 & 1.555 & .892 & 2.712 & & & & \\
\hline Localisation: parenchymal \& meningeal & .504 & .801 & .417 & 1.537 & & & & \\
\hline \multicolumn{9}{|l|}{ TTBM (reference: < 12 months) } \\
\hline TTBM (12-24 months) & .536 & 1.120 & .782 & 1.604 & .934 & 1.017 & .686 & 1.507 \\
\hline TTBM (24-60 months) & .746 & .944 & .667 & 1.336 & .461 & .869 & .598 & 1.263 \\
\hline TTBM > 60 months & .000 & .453 & .299 & .686 & .006 & .526 & .332 & .834 \\
\hline
\end{tabular}

$\mathrm{Cl}$ confidence interval, ECOG Eastern Cooperative Oncology Group, H1-H4 histology groups (see text for description), HR hazard ratio, MVA multivariate analysis, No number, PS performance status, TTBM time to brain metastasis (in months), UVA univariate analysis Entries in bold represent statistically significant values

$(n=14)$ and solitary fibrous tumors (SFT)/hemangiopericytoma $(n=7)$ ], with $\mathrm{H} 4$ having the best prognosis and $\mathrm{H} 1$ the worst; all individual pairwise comparisons showed statistically significant difference. The split level chosen for ECOG PS were $0-1,2$ and 3-4. For the number of CNS metastases, the split levels found to be more informative were $1,2-4$ and $>4$ lesions, differently to the original GPA score.
The log-rank test of the final model and all pairwise comparisons showed a statistically significant difference in median OS between each sarcoma-GPA grouping $(p<0.0001)$ (Fig. 1c). The addition of the variables age, TTBM and presence of ECM in the under construction indices, assessed at several different split-levels, did not improve their prognostic significant, but rather compromised it (data not shown). 


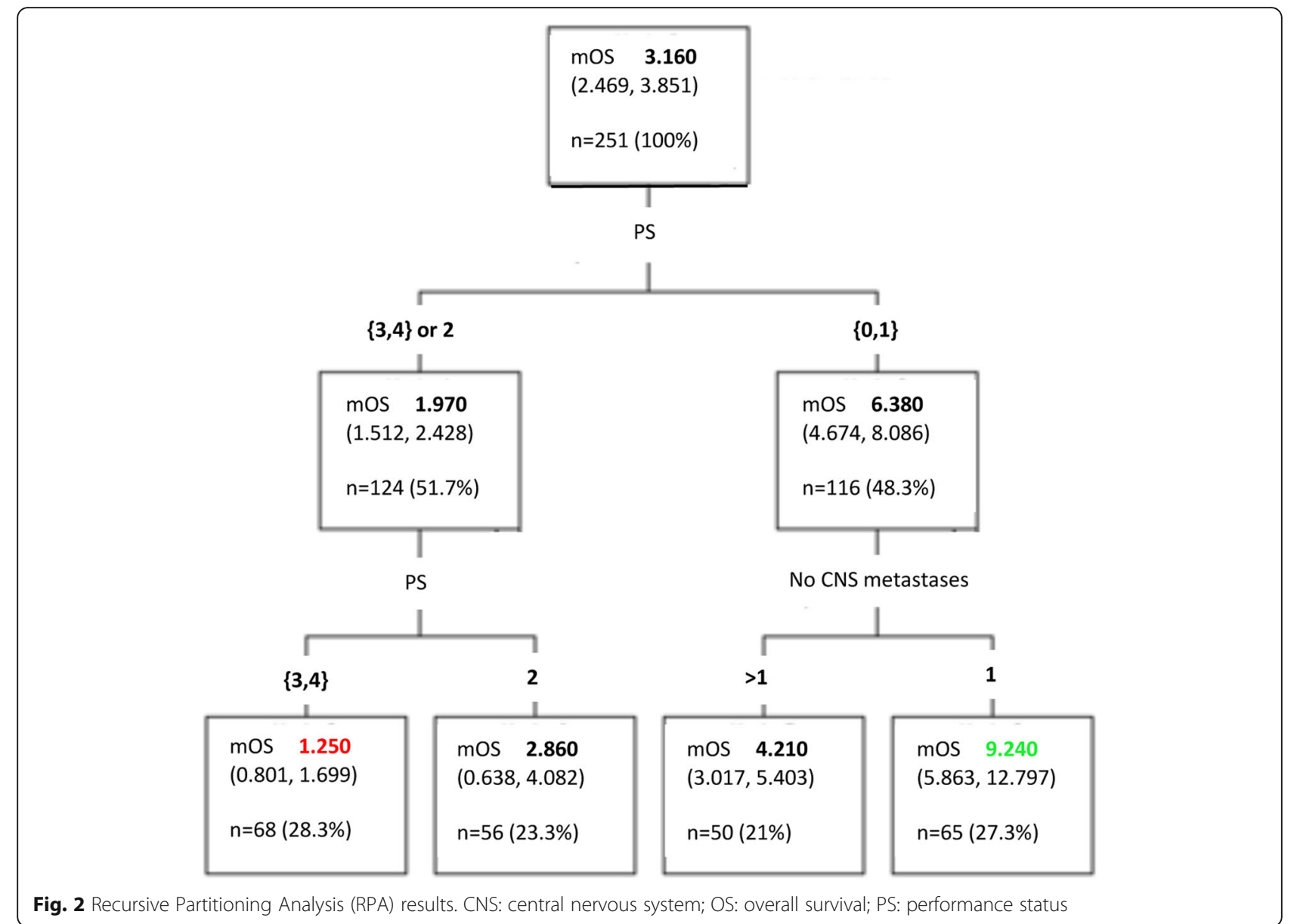

Although both ECOG PS and KPS were individually prognostic (Fig. 2e\&ff), ECOG PS was found to have a better separation power amongst sub-groups within the final index in comparison to KPS (Fig. 3 vs Additional file 5: Figure S3). The c-index for the original GPA was 0.649, which improved to 0.688 using the Sarcoma-GPA. The ROC curves for the original GPA and Sarcoma GPA corroborated the c-index results (data not shown).

As the Sarcoma-GPA index was designed to provide prognostic information independently of treatment modality, the use of the treatment modalities in our patient cohort was assessed for statistically significant difference. Additional file 2: Table S2 shows the distribution of the different treatment modalities in the individual histology sub-groups as defined above for the Sarcoma-GPA index. No statistically significant difference was observed for any of the treatment modalities between histology groups, with the exception of targeted therapy.

We also assessed whether OS was significantly different in the better prognosis groups (H4 and GPA4) according to the different treatment modalities. The differences were not statistically significant, indicating that the use of different treatment modalities did not significantly influence outcome; for the $\mathrm{H} 4$ group the $p$ values were $0.222,0.386,0.019,0.061,1.00$ and 0.37 , whilst for the GPA4 group the p values were 0.048, $0.125,0.048,0.245,0.938$ and 0.107 for the WBRT, SRS, surgery, systemic chemotherapy, targeted therapy and BSC, respectively (not applicable for the intrathecal chemotherapy, as no patient in the H4 or GPA4 groups received this modality). Significance cut-off for the above was the same used for the construction of the SarcomaGPA index, i.e. $p<0.001)$.

\section{Discussion}

Two components of the original GPA index were retained in the Sarcoma-GPA, albeit modified. Notably, the patient general status was included in the final index scored according to the ECOG PS score, as was more informative within the final index compared to KPS. The number of BMs was also retained, however alternative split-levels was found to be more informative within the final index ( 1 vs $2-4$ vs $>4$ lesions) (Fig. 3).

The presence of extracranial metastases, a component of the original GPA index [8] (Table 1), the lung cancerspecific GPA [10], and maintained in the updated Lung- 


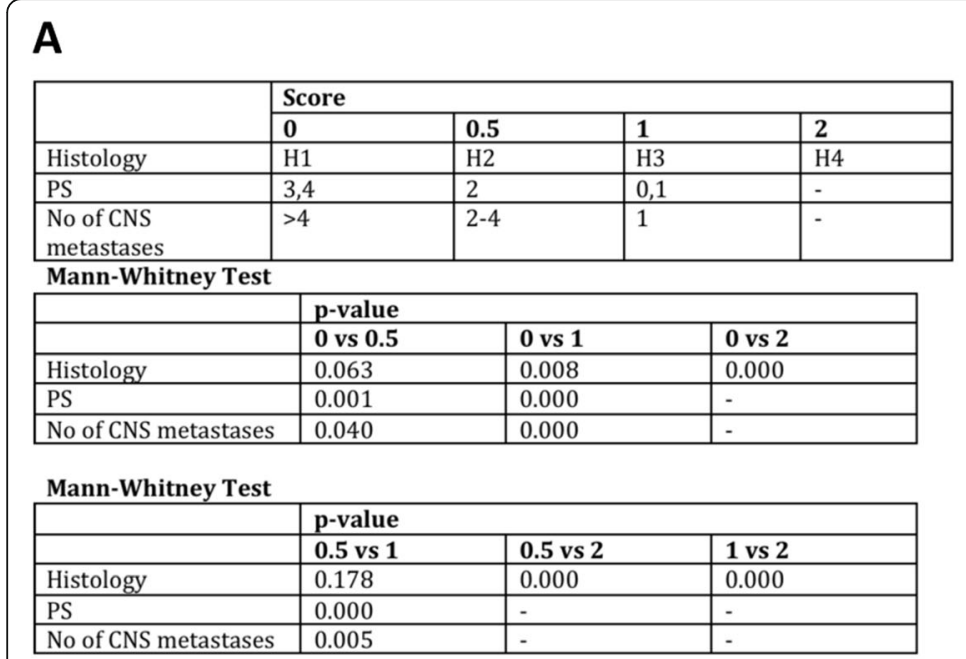

B

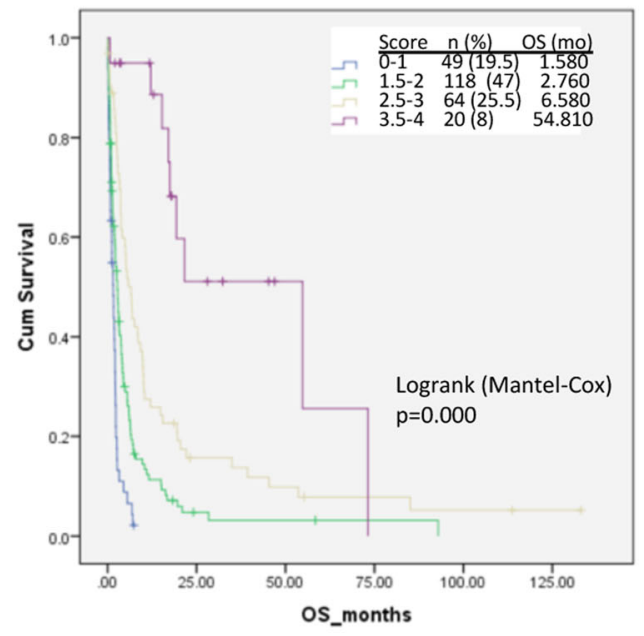

C

Pairwise Comparisons

\begin{tabular}{|l|l|r|r|r|r|r|r|r|r|}
\hline & \multirow{2}{*}{$\begin{array}{l}\text { Sarcoma- } \\
\text { GPA score }\end{array}$} & \multicolumn{2}{|c|}{$0-1$} & \multicolumn{2}{c|}{$1.5-2$} & \multicolumn{2}{c|}{$2.5-3$} & \multicolumn{2}{c|}{$3.5-4$} \\
\cline { 2 - 9 } & Chi-Square & Sig. & Chi-Square & Sig. & Chi-Square & Sig. & Chi-Square & Sig. \\
\hline & $0-1$ & & & 15.290 & .000 & 49.628 & .000 & 43.616 & .000 \\
Log Rank & $1.5-2$ & 15.290 & .000 & & & 13.938 & .000 & 28.808 & .000 \\
(Mantel-Cox) & $2.5-3$ & 49.628 & .000 & 13.938 & .000 & & & 11.649 & .001 \\
& $3.5-4$ & 43.616 & .000 & 28.808 & .000 & 11.649 & .001 & & \\
\hline
\end{tabular}

Fig. 3 Sarcoma Graded Prognostic Assessment (Sarcoma-GPA) index. a. Prognostic factors, point groupings and Mann-Whitney test for significance of split levels; b. Kaplan-Meier curves for overall survival levels by Sarcoma-GPA group; $\mathbf{c}$ : Pairwise comparisons using the Mantel-Cox logrank test for the Sarcoma-GPA groups, demonstrating statistically significant separation between groups. CNS: central nervous system; H1-H4: histology groups (see text for description); OS: overall survival; PS: ECOG performance status

molGPA [13], as well as in the Melanoma-molGPA [14], was not found to be prognostic for sarcoma BMs, potentially as an influence of the predominance of the presence of ECM at the time of sarcoma BM diagnosis, a finding consistent with previous literature [3]. Similarly, age, another original GPA index variable, was not retained as significant despite repeated analyses at different split-levels.

The important addition of the histology in the SarcomaGPA has helped increase the discriminative power of the index and identify a histology subgroup with especially good prognosis (the $\mathrm{H} 4$ histology group, median OS 20.45 months). The final combined index is able to stratify patients with a OS of more or less than 6 months, with two sub-groups on either side of this timepoint. The discriminative power of histological type is not surprising for sarcomas, as they comprise a highly diverse tumour group, with distinctive pathological features and molecular basis, as well as variable prognosis. In this context, the SarcomaGPA index described here is akin to the updated molecular GPA indices for lung cancer and melanoma [13, 14].
In the Sarcoma-GPA, the combination of the H4 histology groups tumors with a limited number of BMs and a good ECOG performance status is able to select for a particularly favorable prognosis group, with an estimated median OS of almost 55 months (Fig. 3).

ASPS is a rare histology, characterised by a specific molecular change $[\mathrm{t}(\mathrm{X} ; 17)(\mathrm{p} 11 ; \mathrm{q} 25)$ translocation, resulting in an ASPL-TFE3 gene fusion] [21], and is known to have an indolent clinical course in the non-metastatic stage, however characterized by late metastases with a 5 -year OS of $20 \%$ at the metastatic stage [22, 23]. ASPS feature a well-established preponderance for BMs, with a reported incidence of approximately $20-35 \%$ [22, 24-28], compared to $<1-8 \%$ of sarcoma patients developing BMs overall $[3,29,30]$. Our study featured a median OS for the ASPS cohort $(n=14)$ of 17.33 months, indicating that a relatively long survival is retained event in the presence of BMs, consistent with previous sporadic reports [27]. In contrast to traditional reports of ASPS as frequently associated with ECM [22, 24], none of our 14 cases were; this might be due to routine brain staging of asymptomatic patients at diagnosis, and also explain the 
relatively long survival, as these patients had relatively lowvolume metastatic disease $(\sim 70 \%$ had a single $\mathrm{BM}$, none had $>4$ lesions, none featured ECM). Our haemangiopericytoma/SFT cases were similarly not associated with ECMs (although they were not primary intracranial meningeal haemangiopericytomas), consistent with the majority of previous literature, which nevertheless is extremely limited [31-34].

The value of our report is highlighted by the difficulty of obtaining large-volume data for BMs of sarcoma patients, given their rarity. The development of the BRAINSARC project was an optimal opportunity to develop a prognostic index for this heterogeneous group of diseases. Within the BRAINSARC project, we had identified a subset of patients with survival longer than 2 years [17]. Histology alone was not able to select for these patients as this group, other than ASPS and SFTs, also included leiomyosarcoma, synovialosarcomas and Ewing/PNET tumors. This is concurrent with our analysis, as the $\mathrm{H} 4$ histology group had a median OS of 20.45 months (Fig. 2), i.e. less than the $>24$ months necessary to be classified as long survivors in our previous report. When, however, the H4 histology group variable was enriched within the overall index by its association with ECOG PS and number of BMs, this became a much more powerful prognostic tool (Fig. 3). Our previous long-survivor analysis, which indicated that long survivors featured a greater percentage of unique BM lesions and better ECOG PS, corroborates this [17].

It should be noted that the construction of the histology sub-groups was based on the split levels indicated by statistical analysis in regard with significantly differential survival, and not selected based on histological lineage, nevertheless a certain lineage coherence is indeed reflected in the H1-H4 grouping (for example, adipocytic tumors in $\mathrm{H} 1$ and musculoskeletal tumors in $\mathrm{H} 2$ ). Once the overall cohort was optimally split in the four survival groups, the histological types comprising these four subgroups were detailed, as described in the Results section. It is therefore a grouping pertaining to the survival of sarcoma BM patients, a meaningful way to segregate how different histologies fare according to BM patient survival, and not a strict histological affinity classification.

Although the choice of treatment modality is beyond the scope of this manuscript, it is important to highlight that his study reports on patients managed over a very large period of time, as was necessary in order to obtain a large enough cohort, owing to the rarity of brain metastases in sarcoma patients. In this period of over 25 years since the beginning of our reporting period, management of metastatic brain disease has enormously evolved, from very conservative and restricted to more aggressive even in the presence of extracranial disease, and this is reflected in the reported treatment modalities. Overall, the use of different treatment modalities, and notably local modalities known to be associated with better outcome in general (surgery, SRS) neither differed nor significantly influenced OS in the histology and GPA groups, which indicates that the better outcome of the H4 and GPA4 group was not influenced by a differential use of treatment modalities in these sub-groups, further strengthening the prognostic value of the index we describe in this paper.

The high spatial and temporal tumoral heterogeneity and clonal shift occurring between primary and metastatic sites poses a complex therapeutic challenge. Brain metastases are a distant reflection of the primary, with the specific peculiarities of the CNS microenvironment. The decision to apply a treatment or not in the presence of BMs is a cardinal one, and precedes the one of the modality choice. The aim of this paper was to derive a purely prognostic index, in order to provide guidance into the decision of treating a patient with sarcoma BM lesion(s). It is constructed on a smaller number of patients than the previous GPA indices, however this needs to be assessed in the context of the relative rarity of the sarcoma BMs. This index does not take into consideration the potential effects of treatment on the patient quality of life, a factor that needs to be evaluated for the final decision-making.

With the increased incidence of BMs in all cancer types and the evolvement of systemic treatment options leading to globally increased cancer survival, it has become crucial to adapt treatment attitudes for the presence of brain lesions, correctly identify patients that merit local treatment, obtain realistic estimates of survival and select for the optimal treatment strategy. This has been an issue ignored for long in the development of new strategies and drug development, but the paradigm has already started to change. Modern strategies for clinical trials not only allow and stratify for the presence of BMs, but trials are also specifically designed for BM patients. Even further, prognostic indices are nowadays incorporated in the design of clinical trials [35].

\section{Conclusions}

The Sarcoma-GPA provides a novel tool to sarcoma oncologists to guide clinical decision-making and outcomes research. Tailored to the specifics of histological variations and characteristics of sarcoma patients with lesions homing to the brain, it identifies favorable prognosis patients that are more likely to gain an enhanced clinical benefit from BM-directed treatment. Prospective independent validation of the described index is needed, and this is currently planned in the context of a multinational project, as the rarity of sarcoma brain metastasis dictates the need for such a collaborative effort. 


\section{Supplementary information}

Supplementary information accompanies this paper at https://doi.org/10. 1186/s12885-020-6548-6.

Additional file 1: Table S1. Treatment modalities. BSC: best supportive care; MD: missing data; SRS: stereotactic radiosurgery; WBRT: whole-brain radiotherapy

Additional file 2: Table S2. Treatment modalities per histology group BSC: best supportive care; SRS: stereotactic radiosurgery; WBRT: wholebrain radiotherapy.

Additional file 3: Figure S1. Application of the original GPA index in the sarcoma patient cohort. A. Prognostic factors, point groupings and Mann-Whitney test for significance of split levels; B. Kaplan-Meier curves for overall survival for the original GPA score; C: Pairwise comparisons for the original GPA index using the Mantel-Cox logrank test, demonstrating insufficient separation between groups in sarcoma patients. CNS: central nervous system; KPS: Karnofsky performance status; OS: overall.

Additional file 4: Figure S2. Original GPA components applied in our sarcoma cohort. A. Age; B. Karnofsky performance status (KPS); C. Number of CNS lesions; D. Presence of extracranial metastases. CNS: central nervous system; OS: overall survival.

Additional file 5: Figure S3. Sarcoma Graded Prognostic Assessment index based on KPS. A: Prognostic factors, point groupings and MannWhitney test for significance of split-levels; B: Kaplan-Meier curves for overall survival levels by Sarcoma-GPA group; C: Pairwise comparisons using the Mantel-Cox logrank test. $\mathrm{H} 1-\mathrm{H} 4$ : histology groups (see text for description). CNS: central nervous system; KPS: Karnofsky performance status; OS: overall survival.

\section{Abbreviations}

BM: Brain metastasis; BSSM: Basic Score for Brain Metastases;

ECM: Extracranial metastases; FNCLCC: Fédération nationale des Centres de lutte contre le cancer; GPA: Graded Prognostic Assessment; GSF-

GETO: Groupe Sarcomes Française - Groupe d'Etudes des Tumeurs Osseuses; KPS: Karnofsky performance status; RPA: Recursive Partitioning Analysis; RTOG: Radiation Therapy Oncology Group

\section{Acknowledgements}

No acknowledgements.

\section{Authors' contributions}

Concept of the study: AP. Design of the study: AP, KK, ALC, FD, RS, PWS. Data collection: AP, LC, NI, IRQ, TV, BM, ML, JOB, LMZ, EB, SPN, NP, TA, CG, FD, AH, CLP, FD, JYB, ALC. Statistical analysis: AP, KK. Interpretation of results: AP, KK, PWS, RS, ALC, JYB. Manuscript preparation: AP. Manuscript revision: AP, LC, $\mathrm{NI}, I R Q$, TV, BM, ML, JOB, LMZ, EB, SPN, NP, TA, CG, FD, AH, CLP, FD, JYB, ALC, $K K, R S, P W S$. Approval of final manuscript: $A P, L C, N I, I R Q, T V, B M, M L, J O B$, LMZ, EB, SPN, NP, TA, CG, FD, AH, CLP, FD, JYB, ALC, KK, RS, PWS.

\section{Funding}

No dedicated funding existed for this work.

\section{Availability of data and materials}

Individual patient data are part of the individual centre clinical databases. Raw data supporting the findings can be requested by contacting the Corresponding author.

\section{Ethics approval and consent to participate}

This retrospective study was approved by the multi-institutional review board of the French Sarcoma Clinical Reference Network NETSARC (website: http://www.netsarc.org). Institutional ethics committee approval was also obtained by the two Principal Investigator centres (IRFC, Besançon, France for LC and Centre François Leclerc, Dijon, France for IN). Written informed consent was obtained from all alive study subjects. The study was performed in accordance with the Declaration of Helsinki.

\section{Consent for publication}

Not applicable

\section{Competing interests}

The authors declare that they have no competing interests.

\section{Author details}

${ }^{1}$ Gustave Roussy Cancer Campus, Villejuif, France. ${ }^{2}$ Present Address: Sarah Cannon Research Institute and UCL Cancer Institute \& University College London Hospitals, 93 Harley Street, London W1G 6AD, UK. ${ }^{3}$ IRFC, Besançon, France. ${ }^{4}$ Centre François Leclerc, Dijon, France. ${ }^{5}$ Democritus University, Orestiada, Greece. ${ }^{6}$ Gamma Knife Center, University of Minnesota, Minneapolis, MN, USA. ${ }^{7}$ Centre Léon Bérard, Lyon, France. ${ }^{8}$ Institut Claudius Regaud, Toulouse, France. ${ }^{9} \mathrm{CHU}$ Tours, Tours, France. ${ }^{10} \mathrm{ICM}$, Montpellier, France. ${ }^{11} \mathrm{CHU}$ Clermont-Ferrand, Clermont-Ferrand, France. ${ }^{12}$ Institut Paoli Calmettes, Marseille, France. ${ }^{13}$ Centre René-Gauducheau, Nantes, France.

${ }^{14}$ Institut Marie Curie, Paris, France. ${ }^{15}$ Centre Oscar Lambret, Lille, France.

${ }^{16}$ McGill University Health Centre, Montreal, Canada. ${ }^{17}$ Centre Henri Becquerel, Rouen, France. ${ }^{18}$ Hôpital La Timone, Marseille, France. ${ }^{19} \mathrm{Geneva}$, Switzerland.

Received: 17 March 2019 Accepted: 16 January 2020

Published online: 12 February 2020

\section{References}

1. Fox BD, Patel A, Suki D, Rao G. Surgical management of metastatic sarcoma to the brain. J Neurosurg. 2009;110(1):181-6.

2. Salvati M, D'Elia A, Frati A, Santoro A. Sarcoma metastatic to the brain: a series of 35 cases and considerations from 27 years of experience. J NeuroOncol. 2010;98(3):373-7.

3. Espat NJ, Bilsky M, Lewis JJ, Leung D, Brennan MF. Soft tissue sarcoma brain metastases. Prevalence in a cohort of 3829 patients. Cancer. 2002;94(10): 2706-11.

4. Gaspar L, Scott C, Rotman M, Asbell S, Phillips T, Wasserman T, et al. Recursive partitioning analysis (RPA) of prognostic factors in three radiation therapy oncology group (RTOG) brain metastases trials. Int J Radiat Oncol Biol Phys. 1997;37:745-51.

5. Gaspar L, Scott C, Murray K, Curran W. Validation of the RTOG recursive partitioning analysis (RPA) classification for brain metastases. Int J Radiat Oncol Biol Phys. 2000;47:1001-6.

6. Weltman E, Salvajoli JV, Brandt RA, de Morais HR, Prisco FE, Cruz JC, et al. Radiosurgery for brain metastases: a score index for predicting prognosis. Int J Radiat Oncol Biol Phys. 2000;46:1155-61.

7. Lorenzoni J, Devriendt D, Massager N, David P, Ruiz S, Vanderlinden B, et al. Radiosurgery for treatment of brain metastases: estimation of patient eligibility using three stratification systems. Int J Radiat Oncol Biol Phys. 2004;60:218-24

8. Sperduto PW, Berkey B, Gaspar LE, Mehta M, Curran W. A new prognostic index and comparison to three other indices for patients with brain metastases: an analysis of 1,960 patients in the RTOG database. Int J Radiat Oncol Biol Phys. 2008;170(2):510-4.

9. Sperduto PW, Chao ST, Sneed PK, Luo X, Suh J, Roberge D, et al. Diagnosisspecific prognostic factors, indexes, and treatment outcomes for patients with newly diagnosed brain metastases: a multi-institutional analysis of 4,259 patients. Int J Radiat Oncol Biol Phys. 2010;77:655-61.

10. Sperduto PW, Kased N, Roberge D, Xu Z, Shanley R, Luo X, et al. Summary report on the graded prognostic assessment: an accurate and facile diagnosis- specific tool to estimate survival for patients with brain metastases. J Clin Oncol. 2012;30(4):419-26.

11. Sperduto PW, Kased N, Roberge D, Xu Z, Shanley R, Luo X, et al. The effect of tumor subtype on survival and the graded prognostic assessment (GPA) for patients with breast cancer and brain metastases. Int J Radiat Oncol Biol Phys. 2012;82(5):2111-7.

12. Sperduto PW, Kased N, Roberge D, Chao ST, Shanley R, Luo X, et al. The effect of tumor subtype on the time from primary diagnosis to development of brain metastases and survival in patients with breast cancer. J Neuro-Oncol. 2013;112:467-72.

13. Sperduto PW, Young TJ, Beal K, Pan H, Brown PD, Bangdiwala A, et al. Estimating survival in patients with lung Cancer and brain metastases. An update of the graded prognostic assessment for lung Cancer using molecular markers (lung-molGPA). JAMA Oncol. 2017;3(6):827-31.

14. Sperduto PW, Jiang W, Brown PD, Brunstein S, Sneed P, Wattson DA, et al. Estimating survival in melanoma patients with brain metastases. An update 
of the graded prognostic assessment for melanoma using molecular markers (melanoma-molGPA). Int J Radiat Oncol Biol Phys. 2017;99(4):812-6.

15. Wu Q, Li J, Zhu S, Wu J, Chen C, Liu Q, et al. Breast cancer subtypes predict the preferential site of distant metastases: a SEER based study. Oncotarget. 2017;8(17):22790-7996

16. Gong Y, Liu YR, Ji P, Hu X, Shao ZM. Impact of molecular subtypes on metastatic breast cancer patients: a SEER population-based study. Sci Rep. 2017;7:45411-20.

17. Chaigneau L, Patrikidou A, Ray-Coquard I, Valentin T, Linassier C, Bay JO, et al. Brain metastases from adult sarcoma: prognostic factors and impact of treatment. A retrospective analysis from the French sarcoma group (GSF/ GETO). Oncologist. 2018;23(8):948-55.

18. Guillou L, Coindre JM, Bonichon F, Nguyen BB, Terrier P, Collin F, et al. Comparative study of the National Cancer Institute and French Federation of Cancer Centers Sarcoma Group grading systems in a population of 410 adult patients with soft tissue sarcoma. J Clin Oncol Off J Am Soc Clin Oncol. 1997;15(1):350-62.

19. Fletcher CDM, Bridge JA, Hogendoorn P, Mertens F. WHO Classification of Tumours of Soft Tissue and Bone. 4th ed. Geneva: WHO; 2013. IARC WHO Classification of Tumours, No 5

20. van Dijk MR, Steyerberg EW, Stenning SP, Dusseldorp E, Habbema JDF. Survival of patients with nonseminomatous germ cell cancer: a review of the IGCC classification by cox regression and recursive partitioning. $\mathrm{Br}$ J Cancer. 2004;90:1176-83.

21. Ladanyi M, Lui MY, Antonescu CR, Krause-Boehm A, Meindl A, Argani P, et al. The $\operatorname{der}(17) t(X ; 17)(p 11 ; q 25)$ of human alveolar soft part sarcoma fuses the TFE3 transcription factor gene to ASPL, a novel gene at $17 \mathrm{q} 25$. Oncogene. 2001;20:48-57.

22. Portera C, Ho V, Patel SR, Hunt KK, Feig BW, Respondek PM, et al. Alveolar soft part sarcoma clinical course and patterns of metastasis in 70 patients treated at a single institution. Cancer. 2001;91:585-91.

23. Lillehei KO, Kleinschmidt-DeMasters B, Mitchell DH, Spector E, Kruse CA. Alveolar soft part sarcoma: an unusually long interval between presentation and brain metastasis. Hum Pathol. 1993:24:1030-4.

24. Lewis AJ. Sarcoma metastatic to the brain. Cancer. 1988;61:593-601.

25. Lieberman PH, Brennan MF, Kimmel M, Erlandson RA, Garin-Chesa P, Fiehinger BY. Alveolar soft-part sarcoma: a clinicopathologic study of half a century. Cancer. 1989;63:1-13.

26. Rosenbaum AE, Gabrielsen TO, Harris H, Goldberg S. Cerebral manifestations of alveolar soft-part sarcoma. Neuroradiology. 1971;99:109-15.

27. Salvati M, Cervoni L, Caruso R, Gagliardi FM, Delfini R. Sarcoma metastatic to the brain: a series of 15 cases. Surg Neurol. 1998:49:441-4.

28. Hoiczyk M, Herbrik M, Grabellus F, Podleska L, Pöttgen C, Schwindenhamme B, et al. Brain metastases in sarcoma patients: Incidence and outcome. J Clin Oncol. 2014;32(suppl; abstr 10591):5s.

29. Bryant BM, Wiltshaw E. Central nervous system involvement in sarcoma. Eur J Cancer. 1980;16:1503-7.

30. Espana $P$, Chang $P$, Wiernik P. Increased incidence of brain metastases in sarcoma patients. Cancer. 1980;45:377-80.

31. Essola B, Remmelink M, Kessler R, Scillia P, Rocmans P. L'hémangiopéricytome pulmonaire primitif: deux nouvelles observations. Rev Med Brux. 2003;24:408-15.

32. Braham J, Sarova-Pinchas I, Pauzner YM, Braf Z. Hemangiopericytoma of the lung with metastasis to the brain. Case report Isr Med J. 1962;21:47-50.

33. Ozeki N, Kawaguchi K, Taniguchi T, Yokoi K. Primary pulmonary solitary fibrous tumour with brain metastases. Eur J Cardio-Thoracic Surg. 2014; 45:386-8.

34. Degnan AJ, Lee KKW, Minervini MI, Borhani AA. Metastatic extrapleural malignant solitary fibrous tumor presenting with hypoglycemia (Doegepotter syndrome). Radiol Case Rep. 2017;12:113-9.

35. White JR, Moughan J, Kim IA, Peereboom DM, De Los Santos JF, Boulter D, et al. NRG/RTOG 1119: Phase II Randomized Study of Whole Brain Radiation Therapy/Stereotactic Radiosurgery in Combination With Concurrent Lapatinib in Patients With Brain Metastasis From HER2-Positive Breast Cancer: A Collaborative Study of NRG Oncology and KROG (NCT01622868). San Antonio Breast Cancer Symposium (SABCS) 2016, abstract OT1-04-02. https://www.sabcs.org/Portals/SABCS2016/Documents/SABCS-2016Abstracts.pdf?v=1.

\section{Publisher's Note}

Springer Nature remains neutral with regard to jurisdictional claims in published maps and institutional affiliations.
Ready to submit your research? Choose BMC and benefit from:

- fast, convenient online submission

- thorough peer review by experienced researchers in your field

- rapid publication on acceptance

- support for research data, including large and complex data types

- gold Open Access which fosters wider collaboration and increased citations

- maximum visibility for your research: over $100 \mathrm{M}$ website views per year

At $\mathrm{BMC}$, research is always in progress.

Learn more biomedcentral.com/submissions 